\title{
Metamagnetism and Fermi Surface in the Anderson Lattice Model
}

\author{
Yoshiaki $\overline{\mathrm{ONO}}$ \\ Department of Physics, Nagoya University, \\ Furo-cho, Chikusa-ku, Nagoya 464-01, Japan
}

\begin{abstract}
We investigate magnetization as functions of external magnetic field $H$ in the $U$-infinite Anderson lattice model within the leading order approximation in the $1 / N$-expansion. At $T=0$, at $H=H_{\mathrm{M}}$ where the Zeeman energy is equal to a certain characteristic energy in the system, the magnetization curve has a kink and the differential susceptibility $\mathrm{d} M / \mathrm{d} H$ shows a jump. At finite temperature, $\mathrm{d} M / \mathrm{d} H$ shows a peak around $H_{\mathrm{M}}$. Its maximum value increases with decreasing $T$ and saturates to a finite value at $T \rightarrow 0$. When $H<H_{\mathrm{M}}$, the $f$ and the conduction electrons form the renormalized bands with a large Fermi surface determined by the Luttinger sum rule. On the other hand, when $H>H_{\mathrm{M}}$, the bands reform themselves significantly free from the Luttinger sum rule, eventually leading to a small Fermi surface at $H \gg H_{\mathrm{M}}$. The results are consistent with the metamagnetic properties observed in the heavy fermion $\mathrm{CeRu}_{2} \mathrm{Si}_{2}$.
\end{abstract}


There have been extensive studies on the metamagnetic behavior observed in the normal metallic states of the heavy fermion compounds. The investigations have been mostly focused on $\mathrm{CeRu}_{2} \mathrm{Si}_{2}$, , 11, 2, 3, 4. whose magnetization shows an abrupt increase with increasing $H$ at a certain critical value, $H=H_{\mathrm{M}} \sim$ 7.7T. Many other properties of the material also show pronounced changes at $H \simeq H_{\mathrm{M}}$. The drastic is the change of the Fermi surface observed in the recent de Haas-van Alphen (dHvA) experiments: [3] the large Fermi surface observed at $H<H_{\mathrm{M}}$ seems to suggest existence of the itinerant $f$-electrons, while the small Fermi surface observed at $H>H_{\mathrm{M}}$ seems to suggest the localized $f$-electrons. Several theoretical studies have been already done on these problems. [5, 6, 6, 8, 9. 10] They comment on the magnetization curves but nothing on the drastic change of the Fermi surface.

The purpose of the present paper is to investigate theoretically the metamagnetic properties including both the magnetization and the Fermi surface. We employ the $U$ - infinite Anderson lattice model in the auxiliary boson representation. 11] Previously, the model has been studied in the limit of zero external magnetic field within the approximation including terms of the leading order in the expansion with respect to the inverse of the spin-orbit degeneracy $(1 / N$-expansion) under the strict local constraints guaranteeing the equivalence of the bosonic version to the original $U$-infinite model. 12 The results very well account for the various properties of the heavy fermion systems in the high temperature incoherent regime exhibiting the Kondo effects, the low temperature coherent regime with heavy fermions and the intermediate temperature regime showing the crossover behavior between the two limiting regimes in a unified way. The present study is a straightforward extension of the previous study to cases with finite external magnetic field.

Our Hamiltonian is given by

$$
\begin{aligned}
\mathcal{H}= & \sum_{m=-J}^{J} \sum_{k_{m}} \epsilon_{k_{m}} c_{k_{m}}^{+} c_{k_{m}}+\sum_{i} \sum_{m=-J}^{J}\left(\epsilon_{f}-m H\right) f_{i m}^{+} f_{i m} \\
& +N_{\mathrm{L}}^{-\frac{1}{2}} \sum_{i} \sum_{m=-J}^{J} \sum_{k_{m}}\left(V_{k_{m}} e^{-\mathrm{i} k_{m} \cdot R_{i}} c_{k_{m}}^{+} f_{i m} b_{i}^{+}+\text {h.c. }\right),
\end{aligned}
$$

where $m$ stands for the degrees of freedom due to the spin-orbital degeneracy, $m=-J,-J+1, \cdots J \quad(2 J+1 \equiv N)$, and $i$ stands for the lattice sites, $i=1,2, \cdots N_{\mathrm{L}} . k_{m}$ represents the generalized wave vector specifying wave vector as well as spin-orbital degrees of freedom. $c_{k_{m}}^{+}$is the creation operator for the $c$-electron. $b_{i}^{+}$and $f_{i m}^{+}$are the creation operators for the slave-boson (SB) and the pseudo-fermion (PF) representing the empty and the singly occupied states of the $i$-th $f$-site, respectively. Here we set $g_{f} \mu_{\mathrm{B}}=1$ and $g_{c} \mu_{\mathrm{B}}=0$. Then, $m H$ is the Zeeman energy for the $f$-electron under the external magnetic field $H$.13 The energies $\epsilon_{k_{m}}$ and $\epsilon_{f}$ are measured relative to the chemical potential $\mu$. 
This model (1) is equivalent to the original $U$-infinite Anderson lattice model as long as it is treated within the physical space where the following local constraints hold:

$$
\hat{Q}_{i} \equiv \sum_{m=-J}^{J} f_{i m}^{+} f_{i m}+b_{i}^{+} b_{i}=1, \quad\left(i=1,2, \cdots N_{\mathrm{L}}\right) .
$$

The expectation value of an operator $\hat{O}$ calculated under the local constraint (2) is given by 11

$$
\langle\hat{O}\rangle=\lim _{\left\{\lambda_{i}\right\} \rightarrow \infty}\left\langle\hat{O} \prod_{i} \hat{Q}_{i}\right\rangle_{\lambda} /\left\langle\prod_{i} \hat{Q}_{i}\right\rangle_{\lambda}
$$

where $\langle\hat{A}\rangle_{\lambda} \equiv \operatorname{Tr}\left[e^{-\beta \mathcal{H}_{\lambda}} \hat{A}\right] / \operatorname{Tr}\left[e^{-\beta \mathcal{H}_{\lambda}}\right]$ with $\mathcal{H}_{\lambda} \equiv \mathcal{H}+\sum_{i} \lambda_{i} \hat{Q}_{i}$. For calculating $\langle\hat{A}\rangle_{\lambda}$, we treat $V_{k_{m}}$ as a perturbation and employ the standard perturbation method using the Feynman diagrams together with the expansion $(1 / N$ expansion) from the large limit of the spin-orbit degeneracy $N$, while keeping the total degrees of freedom for the $c$-electrons to be constant: $N_{\mathrm{L}}^{-1} \sum_{m} \sum_{k_{m}} 1=2$. Following the procedure mentioned above we calculate the single particle Green's functions within the leading order in power of $1 / N$. The explicit forms of the $c$-electron, the SB and the PF Green's functions are given, respectively, by 12

$$
\begin{aligned}
G_{k_{m}}\left(\mathrm{i} \omega_{n}\right) & =\left[\mathrm{i} \omega_{n}-\epsilon_{k_{m}}-\Sigma_{k_{m}}\left(\mathrm{i} \omega_{n}\right)\right]^{-1}, \\
B\left(\mathrm{i} \nu_{n}-\lambda_{i}\right) & =\left[\mathrm{i} \nu_{n}-\lambda_{i}-\sum_{m} \Pi_{m}\left(\mathrm{i} \nu_{n}-\lambda_{i}\right)\right]^{-1}, \\
F_{m}\left(\mathrm{i} \omega_{n}-\lambda_{i}\right) & =\left[\mathrm{i} \omega_{n}-\lambda_{i}-\epsilon_{f}+m H\right]^{-1},
\end{aligned}
$$

where the self-energy parts are given by

$$
\begin{aligned}
& \Sigma_{k_{m}}\left(\mathrm{i} \omega_{n}\right)=\left|V_{k_{m}}\right|^{2} \lim _{\left\{\lambda_{i}\right\} \rightarrow \infty}[-T \\
& \left.\quad \times \sum_{\nu_{n^{\prime}}} F_{m}\left(\mathrm{i} \omega_{n}+\mathrm{i} \nu_{n^{\prime}}-\lambda_{i}\right) B\left(\mathrm{i} \nu_{n^{\prime}}-\lambda_{i}\right) /\left\langle\hat{Q}_{i}\right\rangle_{\lambda}\right], \\
& \Pi_{m}\left(\mathrm{i} \nu_{n}-\lambda_{i}\right)=N_{\mathrm{L}}^{-1} \\
& \quad \times \sum_{k_{m}}\left|V_{k_{m}}\right|^{2} T \sum_{\omega_{n^{\prime}}} F_{m}\left(\mathrm{i} \omega_{n^{\prime}}+\mathrm{i} \nu_{n}-\lambda_{i}\right) G_{k_{m}}\left(\mathrm{i} \omega_{n^{\prime}}\right),
\end{aligned}
$$

with $\left\langle\hat{Q}_{i}\right\rangle_{\lambda} \equiv\left\langle b_{i}^{+} b_{i}\right\rangle_{\lambda}+\sum_{m}\left\langle f_{i m}^{+} f_{i m}\right\rangle_{\lambda}$. The occupation numbers of the SB and the $\mathrm{PF}$ are given in the leading order of the $1 / N$-expansion by

$$
\begin{aligned}
\left\langle b_{i}^{+} b_{i}\right\rangle_{\lambda} & =-T \sum_{\nu_{n}} B\left(\mathrm{i} \nu_{n}-\lambda_{i}\right), \\
\left\langle f_{i m}^{+} f_{i m}\right\rangle_{\lambda} & =T \sum_{\omega_{n}} F_{m}\left(\mathrm{i} \omega_{n}-\lambda_{i}\right) \\
& +T \sum_{\nu_{n}} B\left(\mathrm{i} \nu_{n}-\lambda_{i}\right) \frac{\mathrm{d} \Pi_{m}\left(\mathrm{i} \nu_{n}-\lambda_{i}\right)}{\mathrm{d}\left(\mathrm{i} \nu_{n}\right)} .
\end{aligned}
$$


Note that $F_{m}$ is not modified in the lowest order of $1 / N$ as shown in eq.(6), while the correction of $O(1 / N)$ to $\left\langle f_{i m}^{+} f_{i m}\right\rangle_{\lambda}$ must be included in eq.(10) to obtain $\left\langle\hat{Q}_{i}\right\rangle_{\lambda}$ of $O\left((1 / N)^{0}\right)$. Equations (4i)-(10) constitute a set of self-consistent equations (SCE), which was already solved in the limit of $H=0$ analytically at $T=0$ and numerically at finite temperatures. 12] Now, we solve the SCE with $H \neq 0$.

To begin with, we investigate the magnetization process at $T=0$. With $H \neq 0$, we rewrite the occupation numbers, eqs.(9) and (10), and the sum of them, $\left\langle\hat{Q}_{i}\right\rangle_{\lambda}$, in the low temperature limit as

$$
\begin{aligned}
\left\langle b_{i}^{+} b_{i}\right\rangle_{\lambda} & \stackrel{\lambda_{i} \rightarrow \infty}{\longrightarrow} a \mathrm{e}^{-\beta\left(\lambda_{i}+\epsilon_{f}-E_{0}\right)}, \\
\left\langle f_{i m}^{+} f_{i m}\right\rangle_{\lambda} & \stackrel{\lambda_{i} \rightarrow \infty}{\longrightarrow} \mathrm{e}^{-\beta\left(\lambda_{i}+\epsilon_{f}-m H\right)}+\Delta n_{f m} \mathrm{e}^{-\beta\left(\lambda_{i}+\epsilon_{f}-E_{0}\right)}, \\
\left\langle\hat{Q}_{i}\right\rangle_{\lambda} & \stackrel{\lambda_{i} \rightarrow \infty}{\longrightarrow} \sum_{m} \mathrm{e}^{-\beta\left(\lambda_{i}+\epsilon_{f}-m H\right)}+\mathrm{e}^{-\beta\left(\lambda_{i}+\epsilon_{f}-E_{0}\right)},
\end{aligned}
$$

with $\Delta n_{f m} \equiv-\left.a \frac{\mathrm{d}}{\mathrm{d} \omega} \operatorname{Re} \Pi_{m}(\omega)\right|_{\omega=\epsilon_{f}-E_{0}}$, where $E_{0}$ and $a$ are, respectively, the binding energy and the residue of the resonance state in the SB spectrum, $\operatorname{Im} B\left(\nu-\lambda_{i}+i 0^{+}\right)$, which are determined by the relations:

$$
\begin{aligned}
& \epsilon_{f}-E_{0}-\sum_{m} \operatorname{Re} \Pi_{m}\left(\epsilon_{f}-E_{0}\right)=0, \\
& \frac{1}{a}=1-\left.\sum_{m} \frac{\mathrm{d}}{\mathrm{d} \omega} \operatorname{Re} \Pi_{m}(\omega)\right|_{\omega=\epsilon_{f}-E_{0}} .
\end{aligned}
$$

In the above, both $E_{0}$ and $a$ are functions of $T$ and $H$ to be determined later. $\left.E_{0}^{0} \equiv E_{0}\right|_{T=H=0}$ represents the characteristic energy of the system, which corresponds to the Kondo temperature defined in the single impurity Anderson model. 12] We note that the continuum in the SB spectrum, which has finite intensity for $\nu>\lambda_{i}+\epsilon_{f}-\min \left(E_{0}, J H\right)$, is irrelevant to calculate the occupation numbers at $T=0$. From eq.(3), the average number of the $f$-electron with $m$-th orbit is given by $n_{f m}=\lim _{\lambda_{i} \rightarrow \infty}\left[\left\langle f_{i m}^{+} f_{i m}\right\rangle_{\lambda} /\left\langle\hat{Q}_{i}\right\rangle_{\lambda}\right]$. Using eq.(11), we obtain at $T=0$ as

$$
n_{f m}=\frac{\Delta n_{f m}}{1+\alpha}+n_{f J}^{0} \delta_{m, J},
$$

where $n_{f J}^{0}=\alpha /(1+\alpha)$ is the incoherent part of the $f$-electron with $m=J$, which is due to the zeroth order term w.r.t. $V_{k_{m}}$, and $\alpha \equiv \lim _{T \rightarrow 0} \mathrm{e}^{\beta\left(J H-E_{0}\right)}$. Equation (14) yields the average number, $n_{f}=\sum_{m} n_{f m}$, and the magnetization, $M=\sum_{m} m n_{f m}$, of the $f$-electron, respectively, at $T=0$ :

$$
\begin{aligned}
n_{f} & =(1-a) /(1+\alpha)+n_{f J}^{0}, \\
M & =\sum_{m} m \Delta n_{f m} /(1+\alpha)+J n_{f J}^{0} .
\end{aligned}
$$


Substituting eqs.(5), (6) and (11) into eq.(7), we find

$$
\begin{aligned}
\Sigma_{k_{m}}\left(\mathrm{i} \omega_{n}\right) & =\frac{a\left|V_{k_{m}}\right|^{2}}{\mathrm{i} \omega_{n}-E_{0 m}} \frac{1}{1+\alpha} \\
& -\left|V_{k_{J}}\right|^{2} B\left(\epsilon_{f}-J H-\mathrm{i} \omega_{n}\right) n_{f J}^{0} \delta_{m, J},
\end{aligned}
$$

at $T=0$, with $E_{0 m} \equiv E_{0}-m H$. Substituting eq.(17) to eq.(畂, we obtain the $c$-electron Green's function.

Now we solve eqs.(12) and (13) with eq.(8) at $T=0$ to obtain $E_{0}$ and $a$ together with $\alpha$ for given $H$. Hereafter for the numerical calculations we assume simple $k_{m}$-dependence as: $\epsilon_{k_{m}}=\epsilon_{c}-\xi_{k_{m}}$ and $\left|V_{k_{m}}\right|^{2}=V^{2} \xi_{k_{m}}$ together with a square density of states: $\rho_{m}^{0}(\xi) \equiv N_{\mathrm{L}}^{-1} \sum_{k_{m}} \delta\left(\xi-\xi_{k_{m}}\right)=2 / N$ for $0 \leq \xi \leq 1$, and $\rho_{m}^{0}(\xi)=0$ for otherwise. The parameters are chosen as: $J=\frac{5}{2}(N=$ 6), $n=n_{c}+n_{f}=1.5, \epsilon_{c}-\epsilon_{f}=0.9$ and $V=0.2$. 114

In Fig.1, we show the self-consistent solutions at $T=0$ for $a$ and $\alpha$ as functions of $H$, and those for $E_{0}$ by the dashed line in Fig.2. We find that, at $H<H_{\mathrm{M}}, E_{0}>J H$ and $\alpha=0$, while, at $H>H_{\mathrm{M}}, E_{0}=J H$ and $\alpha \neq 0$, where $H_{\mathrm{M}}=11.12 \times 10^{-4}$ is of order of $E_{0}^{0} / 2 . \quad\left(E_{0}^{0}=22.77 \times 10^{-4}\right.$ as plotted in Fig.2.) Each of $a, \alpha$ and $E_{0}$ has a kink at $H=H_{\mathrm{M}}$. Correspondingly, the magnetization curve has a kink at $H=H_{\mathrm{M}}$ as shown in Fig.3 (see the dashed line for $T=0$ ). At $H=H_{\mathrm{M}}$, the slope of $M$ increases abruptly with increasing $H$, because so does that of the incoherent part $n_{f J}^{0}$ (or $\alpha$ ) as shown in Fig.1 contributing to the 2 nd term in eq.(16).

Next, we investigate the magnetization process at finite temperature. In this case, we can not use the analytic expressions eqs.(14)-(17) obtained at $T=0$. Then we solve the SCE (4)-(10) numerically in the same way as taken in the previous studies. 12] Fig.2 shows $H$-dependence of $E_{0}$ calculated from eq.(12) at several temperatures. When $H>H_{\mathrm{M}}$, we observe that $\left(E_{0}-J H\right) \propto T$ at the low temperatures, and find that the extrapolated value of $\mathrm{e}^{\beta\left(J H-E_{0}\right)}$ at $T \rightarrow 0$ coincides with $\alpha$ calculated through the SCE at $T=0$ (see also Fig.1). In Fig.3, the magnetization curves are plotted at several temperatures. At the lower temperatures, the slope of $M$ increases significantly around $H=H_{\mathrm{M}}$, while, at the higher temperatures, $M$ increases only monotonically. In Fig.4, we plot the differential susceptibility defined by $\mathrm{d} M / \mathrm{d} H$ as functions of $H$ at several temperatures. At the lower temperature $T \lesssim 5 \times 10^{-4}, \mathrm{~d} M / \mathrm{d} H$ shows a peak around $H=H_{\mathrm{M}}$. Its maximum value increases with decreasing $T$ and saturates to a finite value at $T \rightarrow 0$. Such behaviour in $\mathrm{d} M / \mathrm{d} H$ at $T \rightarrow 0$ has been observed in the recent magnetization measurements at very low temperature. 四

The magnetic susceptibility $\chi_{s}$ is plotted as function of $T$ in Fig.5, where $\chi_{s}^{0}=J(J+1) n_{f}^{0} / 3 T$ is the contribution due to the incoherent part of the $f$ electron, $n_{f}^{0}=\sum_{m} n_{f m}^{0}$, while $\Delta \chi_{s}$ is the coherent part due to the lowest order corrections in the $1 / N$-expansion. We observe a maximum in $\chi_{s}$ at $T=T_{\max }$, where $T_{\max } \sim 5.5 \times 10^{-4}$ is of order of $E_{0}^{0} / 4$. At $T \ll T_{\max }, \Delta \chi_{s}$ is the dominant term leading to the enhanced Pauli paramagnetism, $\Delta \chi_{s} \sim 1 / E_{0}^{0}$, 
while, at $T \gg T_{\max }, \chi_{s}^{0}$ is the dominant term leading to the Curie law, $\chi_{s}^{0} \sim$ $J(J+1) / 3 T$.12 At $T \gtrsim T_{\max }$, the incoherent part, $n_{f}^{0}$, contributes dominantly to $M$ even for $H=0$. Thus, the metamagnetic behaviour due to the rapid increase of $n_{f J}^{0}$ is no more distinguished at $T \gtrsim T_{\max }$, as seen in Fig.4 and as observed in the experiments. [1] We emphasize that both $H_{\mathrm{M}}$ and $T_{\max }$ are proportional to $E_{0}^{0}$ which determine the Fermi liquid properties of the system, e.g., $\left.\chi_{s}\right|_{T=0} \sim 1 / E_{0}^{0}$. Such relationship among $H_{\mathrm{M}}, T_{\max }$ and the characteristic energy of the Fermi liquid has been observed in the pressure dependence of metamagnetic properties. [2]

Finally, we discuss the quasi-particle properties and the Fermi surface. Substituting eq.(17) into eq.(11), we obtain the $c$-electron Green's function at $T=0$ for $H<H_{\mathrm{M}}$ as $G_{k_{m}}\left(i \omega_{n}\right)=\sum_{\gamma= \pm} A_{k_{m}}^{\gamma}\left[\mathrm{i} \omega_{n}-E_{k_{m}}^{\gamma}\right]^{-1}$, with

$$
\begin{aligned}
& E_{k_{m}}^{ \pm} \equiv \frac{1}{2}\left[\epsilon_{k_{m}}+E_{0 m} \pm \sqrt{\left(\epsilon_{k_{m}}-E_{0 m}\right)^{2}+4 a\left|V_{k_{m}}\right|^{2}}\right] \\
& A_{k_{m}}^{ \pm} \equiv\left(E_{k_{m}}^{ \pm}-E_{0 m}\right) /\left(E_{k_{m}}^{ \pm}-E_{k_{m}}^{\mp}\right) .
\end{aligned}
$$

In Fig.6(a), $E_{k_{m}}^{ \pm}$are plotted as functions of $\xi_{k_{m}}$ for $H<H_{\mathrm{M}}$, which describe the coherently $c f$-hybridized excitation with the Zeeman split resonance level $E_{0 m}$. On the other hand, for $H>H_{\mathrm{M}}$, the imaginary part of the $c$-electron self-energy with $m=J$ is finite at $\omega<0$ as shown in eq.(17) (note that the continuum in the SB spectra is finite for $\left.\nu>\lambda_{i}+\epsilon_{f}-\min \left(E_{0}, J H\right)\right)$. Therefore the $c f$-hybridized excitation with $m=J$ becomes incoherent. However the incoherent excitation has a finite energy gap as seen in Fig.6(b), because the resonance level $E_{0 J}=0$. Thus they are irrelevant to the low energy properties at $T=0$.

Using eqs.(7) and (10), the average number of the $f$-electron with $m$-th orbit is written by:

$$
n_{f m}=\frac{1}{N_{\mathrm{L}}} \sum_{k_{m}}\left[-T \sum_{\omega_{n}} G_{k_{m}}\left(\mathrm{i} \omega_{n}\right) \frac{\mathrm{d} \Sigma_{k_{m}}\left(\mathrm{i} \omega_{n}\right)}{\mathrm{d}\left(\mathrm{i} \omega_{n}\right)}\right]+n_{f m}^{0} .
$$

Then the total number of the $c$ - and the $f$-electrons per site is given by:

$$
n_{c}+n_{f}=\frac{1}{N_{\mathrm{L}}} \sum_{m, k_{m}}\left[T \sum_{\omega_{n}} \frac{\mathrm{d} \log \left\{G_{k_{m}}\left(\mathrm{i} \omega_{n}\right)^{-1}\right\}}{\mathrm{d}\left(\mathrm{i} \omega_{n}\right)}\right]+n_{f}^{0} .
$$

When $T=0$ and $H<H_{\mathrm{M}}$, it yields $n_{c}+n_{f}=N_{\mathrm{L}}^{-1} \sum_{m} \sum_{k_{m}} \theta\left(-E_{k_{m}}^{-}\right)$, by using $n_{f}^{0}=0$ and $\operatorname{Im} \Sigma_{k_{m}}\left(\mathrm{i} 0_{+}\right)=0$. Thus the Fermi surface is determined by the total number of the $c$ - and the $f$-electrons, leading to a large Fermi surface as seen in Fig.6(a). This is nothing but the Luttinger sum rule.15. On the other hand, when $T=0$ and $H>H_{\mathrm{M}}, n_{f}^{0}=n_{f J}^{0} \neq 0$. Therefore, the Luttinger sum rule breaks down. As $n_{f J}^{0}$ increases with increasing $H$, the phase volume 
enclosed by the Fermi surface with $m \neq J$ decreases abruptly, leading to a small Fermi surface determined almost only by the $c$-electrons as seen in Fig.6(b). The drastic change of the Fermi surface around $H=H_{\mathrm{M}}$ has been observed in the recent $\mathrm{dHvA}$ experiments. [3]

In conclusion, we have investigated the magnetization process of the Anderson lattice model in the metallic phase within the leading order approximation in the $1 / N$-expansion. A critical field $H_{\mathrm{M}}$ has been shown to exist at $T=0$ : at $H>H_{\mathrm{M}}$, the incoherent part of the $f$-electron due to the zeroth order term w.r.t. the $c f$-mixing becomes relevant to the physical quantities, although it is irrelevant at $H<H_{\mathrm{M}}$. Consequently, the magnetization increases far more steeply with increasing $H$ for $H>H_{\mathrm{M}}$, and the differential susceptibility shows a jump at $H=H_{\mathrm{M}}$. Moreover the phase volume enclosed by the Fermi surface has been found to change drastically at $H=H_{\mathrm{M}}$ : at $H<H_{\mathrm{M}}$, it is as large as determined by the Luttinger sum rule, while, at $H>H_{\mathrm{M}}$, it shrinks leading to a small Fermi surface determined almost only by the $c$-electrons. At finite temperature, the smooth metamagnetic behavior has been observed at $T \lesssim T_{\max }$, the temperature where the magnetic susceptibility has a maximum. The results agree with the observation in the metamagnetic heavy fermion $\mathrm{CeRu}_{2} \mathrm{Si}_{2}$.

The author would like to thank Y. Kuroda, T. Matsuura, Y. Ōnuki and T. Sakakibara for useful discussions and comments. This work was partly supported by the Grant-in-Aid for Scientific Research from the Ministry of Education, Science and Culture.

\section{References}

[1] P. Haen, J. Flouquet, F. Lapierre, P. Lejay and G. Remenyi: J. Low. Temp. Phys. 67 (1987) 391.

[2] J. -M. Mignot, J. Flouquet, P. Haen, F. Lapierre, L. Puech and J. Voiron: J. Magn. Magn. Mat. 76\&77 (1988) 97.

[3] H. Aoki, S. Uji, A. K. Albessard and Y. Ōnuki: Phys. Rev. Lett. 71 (1993) 2110; J. Phys. Soc. Jpn. 62 (1993) 3157.

[4] T. Sakakibara, T. Tayama, K. Matsuhira, H. Amitsuka, K. Maezawa and Y. Ōnuki: Phys. Rev. B51 (1995) 1.

[5] W. Brenig: Solid State Commun. 64 (1987) 203.

[6] F. Ohkawa: Solid State Commun. 71 (1989) 907.

[7] R. Konno: J. Phys. Cond. Mat. 3 (1991) 9915.

[8] K. Miyake and Y. Kuramoto: Physica B171 (1991) 20.

[9] S. M. M. Evans: J. Magn. Magn. Mat. 108 (1992) 135. 
[10] T. Saso and T. Hayashi: J. Phys. Soc. Jpn. 63 (1994) 401.

[11] P. Coleman: Phys. Rev. B29 (1984) 3035.

[12] Y. Ōno, T. Matsuura and Y. Kuroda: Physica C159 (1989) 878; J. Phys. Soc. Jpn. 60 (1991) 3475; ibid 63 (1994) 1406; ibid 64 (1995) 1595.

[13] If we assume $g_{c}=g_{f}=g$, we obtain almost the same results for the present case, because the magnetization for the $c$-electron $M_{c}$ is much smaller than that for the $f$-electron $M_{f}: M_{c} / M_{f} \sim O\left(E_{0}^{2} / a V^{2}\right) \ll 1$. 12]

[14] We also examine the other sets of parameters and obtain the same results in the metallic case by rescaling the energies in unit of $E_{0}^{0}$ calculated for each parameter set.

[15] J. M. Luttinger: Phys. Rev. 119 (1960) 1153.

\section{Figure Captions}

Fig.1 Self-consistent solutions for the residue $a$ of the resonance level of the slave-boson (dotted line) and $\alpha$ defined in eq.(14) (solid line) at $T=0$ as functions of $H$.

Fig.2 Self-consistent solutions for the binding energy $E_{0}$ of the resonance level of the slave-boson at $T=0$ (dotted line) and at several finite temperatures (solid lines) as functions of $H$.

Fig.3 Magnetization $M$ at $T=0$ (dotted line) and at several finite temperatures (solid lines) as functions of $H$.

Fig.4 Differential susceptibility $\mathrm{d} M / \mathrm{d} H$ at $T=0$ (dotted line) and at several finite temperatures (solid lines) as functions of $H$.

Fig.5 Temperature dependence of the magnetic susceptibility. The dashed line is the contribution of the incoherent part of the $f$-electron, $\chi_{s}^{0}$, the dotted line is that of the coherent part of $O\left((1 / N)^{0}\right), \Delta \chi_{s}$, and the solid line is the sum of them, $\chi_{s}$.

Fig.6 Schematic structures of the renormalized bands $E_{k}^{ \pm}$for $m=-J,-J+$ $1, \cdots J$ at $H<H_{\mathrm{M}}$ (a) and $H>H_{\mathrm{M}}$ (b) as functions of $\xi_{k}$. The dashed lines in (b) are for $m=J$. The bare $c$-electron band $\epsilon_{k}$ is also plotted (dotted line). 